\title{
EXPLORING THE DOMAIN OF THE CEREBELLAR TIMING SYSTEM
}

\author{
SEAN CLARKE, RICHARD IVRY, JACK GRINBAND, SETH \\ ROBERTS and NAOMI SHIMIZU \\ Department of Psychology. University of California. Berkeley. CA 94720
}

\begin{abstract}
The ability of an animal to process temporal information has adaptive significance across different temporal ranges. The ability to encode and utilize temporal information allows an animal to predict and anticipate events. However, the time scales vary widely. The predictable event might be based on information that changes over relatively long periods such as a year or a day. or over periods comprising much shorter durations. events that change within a few minutes or milliseconds. Are there a single set of neural mechanisms that are essential for representing temporal information over these different scales? Despite the fact that numcrous neural structures have been linked to successful performance on a variety of timing tasks, this question has received relatively little attention. In this chapter. we will focus on the role of the cerebellum in a variety of timing tasks. We will review the hypothesis that the cerebellum can be conceptualized as a relatively task-independent timing mechanism. An important feature of this hypothesis is that the range of the cerebellar timing system is assumed to be relatively restricted. Specifically, we assume that the cerebellum is capable of representing temporal information ranging from a few milliseconds to an upper bound of a few scconds. What remains unclear is whether the cerebellum is involved on tasks spanning longer durations. Cognitive processes such as attention and memory become clearly important here. and indeed. may dominate performance for longer intervals. The animal literature points to noncerebellar structures as playing a critical role in these tasks and we will provide a brief review of this work. Finally. we will present the preliminary results from two experiments designed to directly icst the hypothesis that the cercbellum's temporal capabilities are limited to relatively short durations.
\end{abstract}

\section{Studies with patients with cerebellar lesions}

Ivry and Keele (1989) assessed the performance of a variety of neurological patients and age-matched control subjects on two tasks that were designed to 
require the explicit representation of temporal information. For the time production task, the participants produced a series of simple keypresses, attempting to produce isochronous intervals between each pair of keypresses. For the time perception task, the participants judged whether a comparison interval was shorter or longer than a standard interval. There were three primary groups of paticnts: those with cerebellar lesions, those with Parkinson's disease which would indicate basal ganglia pathology, and those with cortical lesions encompassing premotor regions. Anatomical models as well as consideration of the symptoms associated with cercbellar lesions prompted the inclusion of the first group. The basal ganglia and cortical groups were included both for comparison purposes and because of earlier neuropsychological research implicating basal ganglia (Wing et al., 1984) or frontal/temporal regions (Milner. 1971) in time production or perception. In terms of variability on the repetitive tapping task, the patients with Parkinson's Disease performed comparably to age-matched control subjects. Surprisingly. these null results were obtained under both the on and off medication state. In contrast. patients with either cortical or cerebellar lesions were found to have increased variability on the repetitive tapping task. The total variability was decomposed into two components. that associated with central control processes and that associated with motor implementation (Wing and Kristofferson. 1973). From this analysis. the patients' deficits were attributed to both sources. However. a second study focused on patients with unilateral cerebellar lesions, either in medial or lateral regions. Here, a double dissociation was obtained. Whereas medial lesions led to increased implementation variability, lateral lesions led to an increase in central variability (Ivry et al.. 1988). This dissociation is in accord with neuroanatomical models which emphasize ascending projections from the lateral cerebellum and descending projections from the medial cerebellum. From the tapping results. it is not easy to determine whether the cerebellum is critical for regulating timing. or some other aspect of motor performance.

Whilc Wing and Kristofferson (1973) labeled the central component. "clock variability", this component actually includes all sources of variability not included in the estimate of motor implementation variability (Ivry and Hazeltine, 1995). For this reason, the perception task provides an opportunity to determine whether a particular structure was essential for internal timing. Corrclational studies have suggested that a common mechanism is invoked in both motor and perceptual timing (Keele et al., 
1985). From this, we might expect to find that lesions of a particular brain region will impair performance on both time production and perception tasks. Only the patients with cerebellar lesions showed this dual-deficit. They were significantly impaired on the time perception task, requiring a larger difference between the comparison and standard intervals in order to achieve a criterion level of performance. The perceptual deficit was specific for time discrimination in that the cerebellar patients were unimpaired on an intensity discrimination task. Importantly. the cortical group was normal on the time perception task. but impaired on the intensity task. Thus, the perception task provided a second double dissociation suggesting a special role for the cerebellum in both motor and perceptual tasks that require precise timing. As with the tapping results, the Parkinson patients performed within normal bounds on the time perception task.

These results required a reconceptualization of the domain of cerebellar function. This structure has generally been linked to motor functions. or sensorimotor learning. The fact that the patients were impaired on a purely perceptual task suggested that its domain should be specified in terms of a particular mental operation. namely the representation of the temporal relationships between events. We have hypothesized that this computational capability is invoked across a wide range of tasks that require this form of representation. For examplc. Ivry and Diener (1991) reported that cerebellar patients were impaired on a velocity perception task and that this perceptual problem could not be attributed to a problem in occulomotor control. Indeed. they proposed that some of the eve movement problems observed following cerebellar lesions may reflect an inability to represent the metrical properties of a moving stimulus

\section{The cerebellum and sensorimotor learning}

Impressive progress has been made over the past few decades towards identifying the neural structures involved with different forms of learning and memory. Given the obvious advantages imposed by learning, it is not surprising that a large number of neural structures have been implicated in these processes. An important endeavor has been to specify the domain of these structures and develop computational models to explain their contributions. 
One approach for undurstanding the computational requirements of different learning situations is to consider the temporal properties imposed by different tasks. For example, the learning process in classical conditioning is constrained by the temporal relationship between the CS and US (see Flaherty, 1985; Jenkins, 1984). A prerequisite for learning across a wide range of paradigms is that the onset of the CS precede the US. The most effective inter-stimulus interval (ISI), however, varies depending on the type of learning. Three general categories can be described. 1) Conditioning of simple skeletal reflexes such as the eycblink reflex is limited to short ISIs. being strongest when this interval is less than 1 sec. 2) Conditioning of autonomic responses such as heart-rate conditioning can occur with these short ISIs, but can also be robust when the ISI is extended to the minutes range. 3) Conditioning of avoidance behavior such as in food aversion experiments can be found when the CS and US are separated by durations up to many hours. Moreover. whereas the pairing of a CS and US may lead to multiple CRs, the timing of these learned responses can be quite different.

Conditioning of the rabbit nictitating membrane response (NMR) has become a model paradigm for investigating the neural substrates of basic associative learning and memory processes associated with simple skelctal reflex responses. In NMR conditioning. a neutral CS such as a tone or light is paired with an aversive US (c.g.. an airpuff directed near the eye). After relatively few presentations. the animal begins to extend the membrane in response to the CS alone. The rate of NMR learning is highly dependent on the ISI. Smith (1968) reported that an ISI of $200 \mathrm{~ms}$ produced the highest percentage of CRs in comparison to ISIs of 100,400, and $800 \mathrm{~ms}$ (see also. Steinmetz, 1990). Few CRs were observed with ISIs of $50 \mathrm{~ms}$. Conditioned NMRs can be found with longer intervals. although the rate and efficacy of learning are reduced. In addition. the topography of the CR is highly constrained by the ISI. The maximum extension of the nictitating membrane occurs just prior to the presentation of the US. Indeed, it is this feature that makes this CR highly adaptive. It permits the organism to attenuate the aversive effects of the US. The importance of timing in NMR conditioning was made clear by the work of Kehoe et al. (1989). When rabbits were conditioned simultaneously with two ISIs. they produced two CRs, each one timed to be maximal just prior to the onset of the upcoming US.

Learning related changes during NMR conditioning have been observed in neurons in several brain regions. including the hippocampus (Berger and Thompson, 1978) and the cerebellum (McCormick and Thompson. 1984). 
However, lesion studies have provided compelling evidence that the cerebellum is essential for NMR conditioning. The exact site of plasticity within the cerebellum has been a source of controversy. Thompson (1986) has argued that the critical locus is the interpositus nucleus, whereas other studies have focused on the importance of the cerebellar cortex (e.g. Yeo et al., 1984). Given that the principal cerebellar inputs, the mossy and climbing fibers, innervate both sites, it is reasonable to assume that learning-related changes may occur in both sites (Perret et al., 1993). If this is so. then we want to consider potential computational differences between nuclear and cortical learning. One possibility is that nuclear mechanisms might support a basic associative process for the formation of a CR. while changes in the cerebellar cortex are essential for shaping the topography of the CR. That is. the precise timing of the $C R$ may result from changes in the cerebellar cortex. This hypothesis is supported by the findings that lesions of the cerebellar cortex disnupt the timing of the CR (Perrett et al., 1993).

It remains difficult to specify the learning domain of the cerebellum (see Ivry, 1993). One possibility is that this structure is essential for forming sensorimotor associations that result in skeletal responses to avoid aversive stimuli (Thompson. 1990). This hypothesis emphasizes the task domain of the cerebellum and focuses on the fact that the climbing fiber pathway provides a salient error signal for shaping appropriate skeletal responses. An alternative hypothesis is that the domain of cerebellar learning extends to those situations in which the animal must precisely represent temporal information. By this way of thinking. the cerebellum is associated with NMR conditioning because this type of learning is only adaptive if it is appropriately timed (Kecle and Ivry. 1991). That is, learning an association and forming the temporal representation of that association can not be thought of as distinct. Of course. other types of associations may not have the same temporal requirements and. as such. would not be expected to be dependent on the cerebcllum. For example, the timing of conditioned autonomic responses in the NMR paradigm seems to be relatively independent of the ISI and this form of learning is unaffected by cerebcllar lesions, even when the NM response itself is abolished (Lavond et al., 1984).

Buonomano and Mauk (1994) have presented a computational model of the cerebellum that produces the associations seen in NMR conditioning as well as the precise topography of the CR (see also, Bullock et al.. 1994). This model does not depend on delay lines or arrays of oscillators, but rather emphasizes known anatomical and physiological properties of this neural 
structure. For example, an input pattern across a set of mossy fibers will trigger dynamic changes in the response properties of many cortical cells. Some of these changes will occur rapidly, e.g., within a few milliseconds. However, due to negative feedback loops and physiological processes such as slow IPSPs, other consequences of the mossy fiber activity may not be evident for hundreds of milliseconds. Thus, the cerebellar cortex can maintain a representation for an extended period of time after the onset of a CS. Coupled with an appropriate learning signal such as the complex burst of the climbing fiber, the system can learn to produce a response at a desired point in time.

\section{Potential limitations of cerebellar timing}

In the Buonomano and Mauk (1994) model, the timing of a particular interval reflects the real-time properties of a set of neural elements. For example, activation over one set of units would correspond to a duration 250 $\mathrm{ms}$ and activation over a different set of units would correspond to a duration of $500 \mathrm{~ms}$. There is no fundamental temporal unit from which larger scale durations are constructed via an oscillatory process. That is. the $500 \mathrm{~ms}$ duration is not created by two cycles through a $250 \mathrm{~ms}$ circuit (or multiple cycles of some fundamental period). Oscillatory models have dominated the psychological and neural literature for a variety of reasons. Oscillation is ubiquitous in biological systems, both at the neural level and in behavior. An appealing feature of oscillatory models is that a simple set of mechanisms can provide temporal processing over a wide range of durations since long temporal intervals can be created via multiple cycles of the fundamental period. Non-oscillatory timing mechanisms do not have this feature. They would be expected to have an upper bound on their temporal range, a duration corresponding to the maximum duration that can be supported by the patterns of connectivity in the network. Longer durations might be represented by joining a real-time network with a memory process. While this has some resemblance to standard clock-counter models, it differs in that there is no basic oscillatory.

We propose that the ccrebellum is best characterized as a non-oscillatory timing system. At present. this hypothesis is best viewed as a conjecture. an idea intended to generate empirical tests. There are a few reasons to suspect an upper bound on a short range timing system. First, as noted above. 
conditioning of skeletal responses such as the NMR are difficult to obtain with ISIs longer than 1-2 sec. Whilc the effects of cerebellar lesions on conditioning with long ISIs has not been established, it does appear that the hippocampus becomes relatively morc important under such conditions (Berger et al., 1986). One possibility is that when the ISI exceeds the cerebellar range, learning shifts to new neural sites. Alternatively, memory capabilities of the hippocampus may become combined with timing capabilities of the cerebcllum. cspecially in trace conditioning paradigms.

Second. behavioral studies in humans suggest that there may be a qualitative change around $2-4 \mathrm{sec}$ in our capacity to represent temporal information. Below this duration, successive events are seen as belonging to a common temporally-defined group. regardless of whether this group has a rhythmic structure or lacks such organization. Above this duration, events are perceived as temporally isolated events, even if they occur periodically (Fraisse. 1963: Povel. 1981). Psychophysical studies have also indicated an increase in the Weber fraction on duration discrimination tasks for intervals longer than $2 \mathrm{sec}$ (Getty. 1975: but sec Allan and Gibbon. 1991). On the motor side. Mates et al. (1994) have shown that when tapping with a periodic pacing signal. people shift from a predictive to an reactive mode as the target interval becomes longer. For intervals less than 2-3 sec. the subjects' responses tended to anticipate the toncs. However, for longer intervals, the responses almost always followed the tones, and indeed. occurred with a latency suggestive of a simple reaction time.

\section{Animal models of temporal discrimination}

Several operant learning paradigms have been developed for exploring how animals process and discriminate temporal information. These tasks typically involve durations spanning from a few seconds (e.g., Allan and Gibbon. 1991) to many seconds (Roberts. 1981: Meck and Church. 1987). This work has led to rigorous theoretical models that succeed in accounting for a wide range of phenomena. Moreover, physiological and pharmacological manipulations have helped identify some of the neural mechanisms associated with the component parts of the models. One popular method has been the peak procedure (c.g. Roberts. 1981: Meck, 1991). In this task. a signal is presented and an animal is provided a reinforcement for the first response emitted after a criterion period of time has elapsed. Under 
such conditions, the aninal will increase its response rate as the target duration is approached. By not providing a reinforcement of a certain percentage of trials, the researcher can observe how the animal's response rate decreases once the target duration has elapsed.

Performance on this and related tasks have generally been evaluated within the context of an information processing model that includes timing, attentional, memory, and dicision processes (e.g., Gibbon and Church, 1984). In this model the clock is conceptualized as a pacemaker, emitting pulses as a Poisson process. These pulses are gated by a switch into an accumulator, or counter process. The state of the gate may be under the influence of attention. The current value of the counter constitutes working memory and is compared to a target duration in reference memory, a trace formed from previously encoded time values. The final processing stage entails a decision process in which the two memory values are compared to determine whether a threshold value has been exceeded, thus triggering a response.

Over the past decadc. a number of studies have been designed to identify the critical neural mechanisms associated with the psychological constructs. For example, Meck (1983) argued for a dissociation of clock and memory processes based on the differential effects of pharmacological agents. Dopaminergic agents caused a transient shift in the peak time. a result consistent with the hypothesis that these drugs affected the speed of the clock. In contrast. neuropeptide and cholinergic agents caused permanent changes in the psychometric functions, indicating that their effect was on reference memory. Based on a lesion study. Olton et al. (1988) concluded that attentional processes were localized to the frontal cortex areas and subcortical structures that project to this region. Lesions of either the frontal cortex or of the nucleus basalis magnocellularis disrupted performance when rats had to time two simultancous stimuli without affecting the processing of either signal when presented individually. Lesions of the medial septal area and fimbria fornix did not disnupt performance on the simultaneous discrimination task, but did disrupt performance when a gap was inserted during the stimulus presentation. This result is consistent with a deficit in working memory, implicating a hippocampal role in this process.

In summary, these studies have emphasized that performance on temporal discrimination tasks involve a complex network that includes limbic, basal ganglia. and frontal structures. Implicit in this work has been the assumption that the same mechanisms will be invoked over a wide range of durations 
(e.g., Nichelli, 1993). For example. a common pacemaker will be triggered by stimuli of varying durations, with a critical difference being the number of counts that accumulate during the extent of a particular stimulus. Indecd, the speed of the pacemaker may vary, and its output is subject to attentional limitations. Thus, while there is a clock-like process, the representation of temporal information is influenced by a number of non-temporal. cognitive processes.

To date, this animal rescarch has ignored the cerebellum, perhaps because this structure has been assumed to be limited to the motor domain while these tasks focus on perceptual and memory processes. Moreover. while some studies have used stimuli that are less than $1 \mathrm{sec}$ (Allan and Gibbon, 1991), the majority of this work has involved stimuli that are considerably longer. frequently ranging up to $40 \mathrm{sec}$. As noted above. we have hypothesized that the cercbellar timing system is limited to relatively short durations. Our working model is that this timing process is relatively immune to cognitive influences. The onset of a stimulus may automatically activate different sets of neurons, and memory demands are minimal. Units active at the offset of the stimulus may become associated with certain responses, a model that does not have the working memory requirements of a counter or require that a current representation be compared to a reference memory.

\subsection{EXPERIMENT 1}

The first experiment was designed to test two hypotheses. First, we wanted to develop an animal model to explore the cerebellar timing hypothesis. Rats were trained to discriminate intervals that ranged from 200 to $850 \mathrm{~ms}$. The effects of cerebellar lesions on their performance was assessed. Based on our human studies. it was predicted that these lesions would increase variability on this task without producing a change in bias. That is. the lesions would increase the noise in the cerebellar timing network without producing an overall change in clock speed (since there is no fundamental timing unit). Second. we also trained the animals on a second duration discrimination task. but here the durations spanned $15-45 \mathrm{sec}$. Based on our conjecture that the cercbellar timing system is limited to relatively short durations. we did not expect lesions of this structure to impair performance on this second task. Evidence of a selective deficit on the short range timing 
task would provide initial cvidence for a dissociation of the mechanisms involved in the representation of short and long range durations.

Our human studies (Ivry and Keele, 1989: Ivry and Diener, 1991) have indicated that the neocercbellum is critical for representing temporal information. The output of the neocerebellum is primarily projected via the lateral nuclei, the dentate and interpositus (composed of the globuse and empoliform in humans). Thus. lesions were targeted to center on the dentate nucleus, with the expectation that the damage would extend to the interpositus nuclei.

4.1.1. Method. Subjects. Twelve naive rats (Fisher 344. Charles River Laboratories. Wilmington. MA) were tested. One rat became sick during the course of the experiment and had to be euthanized. The animals were approximately 90 days old at the beginning of training and were maintained at $80 \%$ of their free feeding weight throughout the experiment. Rats were housed individually in plexiglas cages with water available ad libitum.

Apparatus. Subjects were trained individually in standard operant boxes. each enclosed in a sound attenuating clamber. The front wall of the box contained a recessed food tray and two retractable levers, one on each side of the food tray. Water was available ad libitum on the back wall of the box. Visual stimuli were displayed on circular light panels positioned above each lever and via a houselight situated at the upper left hand comer of the front wall. The presentation of the stimuli. response collection, and reinforcement delivery were controlled by a PC computer. Timing was accurate to .01 milliseconds.

Surgery. All surgical procedures were completed under ascptic conditions. After anesthesia with sodium pentobarbital $(50 \mathrm{mg} / \mathrm{kg})$, rats were placed in a stereotaxic apparatus. The skull surface was exposed and two small holes were made over the dentate nucleus on each side. An electrode was lowered into the holes on cither side and a lesion was made by passing $50 \mathrm{~mA}$ at constant current for 20 seconds. Based on the Paxinos and Watson (1986) atlas, the targeted coordinates for the lesions were +/- 3.4 ML. 6.2 DV, and -11.3 AP from Bregma. Eight randomly selected rats received bilateral lesions in this manner. Three animals were given sham lesions by exposing the dura without lowering the clectrodes or passing any current. Rats were allowed I week to recover. at which time test trials were begun.

Procedure. Animals were tested on a daily schedule in two groups of six. Each session lasted four hours during the training phase and seven hours 
during the test phase. On alternate days, rats were tested on the short range and long range timing tasks. For half of the rats, the overhead light was used for the short range (SR) task and both circular disks were used for the long range (LR) task. The two stimuli were reversed for the other group. For the short range task, the initial durations for the training phase were 300 and $1200 \mathrm{~ms}$. For the long range task, the initial durations were 20 and $40 \mathrm{sec}$. Filled intervals were used: the stimulus was present for its entire duration.

On each trial, one of the two possible stimuli for that session (SR or LR durations) was presented. $100 \mathrm{~ms}$ after the offset of the stimuli, both levers were extended. Following a response or a six sec interval, the levers were retracted. Correct responses were rewarded with a $45 \mathrm{mg}$ sucrose pellet. The mapping of stimuli to the response levers was counterbalanced, with one mapping used for three of the boxes and the reverse mapping used for the other three boxes. For all of the rats. the same mapping was used for both the SR and LR tasks (e.g., the $300 \mathrm{~ms}$ and $20 \mathrm{sec}$ were both associated with the right lever). The inter-trial interval was $30 \mathrm{sec}$. A session consisted of approximately 750 trials for the SR task and 375 trials for the LR task.

Performance with the training values asymptoted at around $90 \%$ correct after 24 sessions (12/task). At this point, the stimulus durations were adjusted to 300 and $750 \mathrm{~ms}$ for the SR task and 25 and $40 \mathrm{sec}$ for the LR task. After another 14 sessions. the animals' performance had returned close to the $90 \%$ asy mptotic valuc.

To obtain psychometric functions. the test phase included both the training durations and probe durations. For the SR task, there were nine probe durations ranging from 200 to $850 \mathrm{~ms}$, with seven of these durations falling between the endpoint values. For the LR task. the nine probe durations ranged from 20 to $45 \mathrm{sec}$. On $50 \%$ of the trials, one of the two training durations was selected and correct responses were reinforced. On the other $50 \%$ of the trials. one of the nine probe durations was selected and no reward was possible. As in the end of the training phase, the tasks alternated by session. The rats completed 36 sessions of the test phase prior to surgery. After a one-weck recovery period, post-surgery testing commenced in the same manner and continued for an additional 23 sessions.

4.1.2. Resulls And Discussion. Figure 1 presents the psychometric functions for the two tasks. In each figure. the probability that the animals respond long is graphed as a function of duration. Only the trials on which a response was made were included. Panels a and $\mathrm{c}$ are the averaged data for 
the lesion and sham groups on the SR task; panels $b$ and $d$ are for the LR task. The data are based on the last 15 presurgery sessions and the first 15 postsurgery sessions. The point of subjective equality (PSE) and standard deviation were estimated from these functions. The first measure is an indicator of bias. It corresponds to the duration at which the group will respond long on $50 \%$ of the trials. Note that an increase in PSE occurs when the probability of responding long decreases. The second measure is one

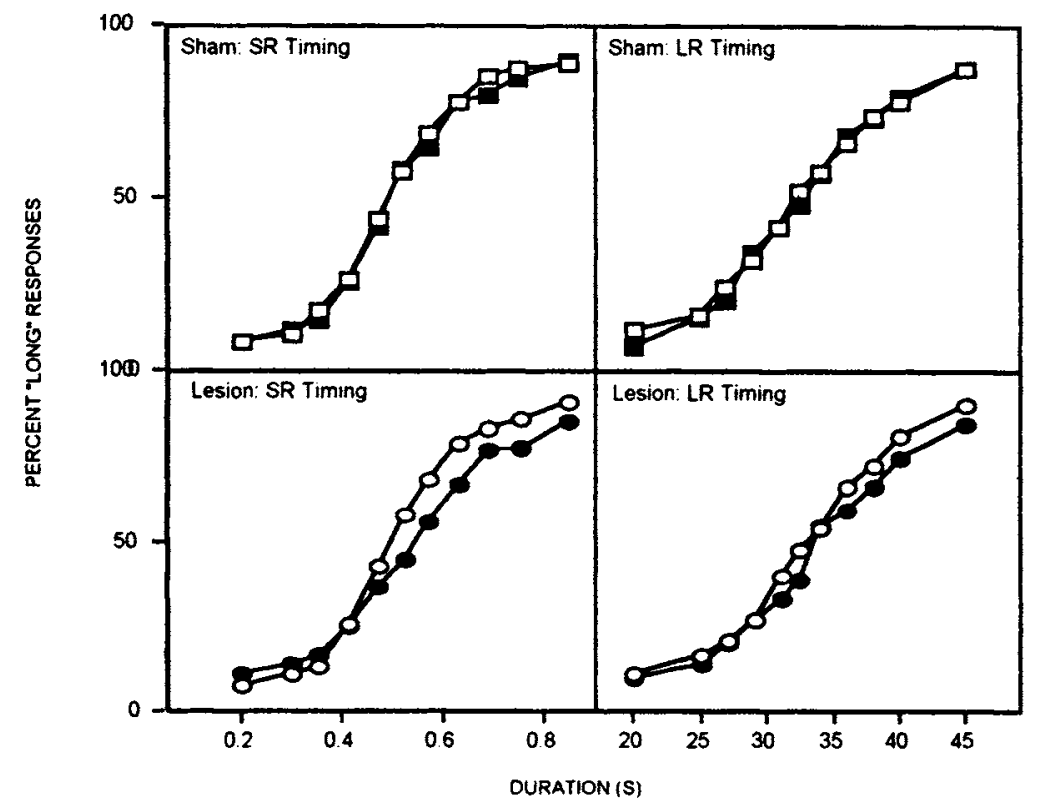

Figure 1: Psychometric functions for Experiment 1. Unfilled symbols represent presurgery performance. filled symbols represent postsurgery performance.

estimate of a difference threshold. or acuity. Steep functions yield low scores indicating that the animals consistently identify a particular stimulus as short or long. Shallow functions yield high scores, indicating more uncertainty in the response functions.

The sham animals showed little change in performance on the SR task following surgery. Their PSEs were $533 \mathrm{~ms}$ and $539 \mathrm{~ms}$ for the pre- and post-surgery sessions. They also showed a small increase in variability, with the standard deviation score rising to $201 \mathrm{~ms}$ from $183 \mathrm{~ms}(9.8 \%)$. In 
contrast, the group receiving cercbellar lesions showed significant changes in performance following surgery. In terms of the aggregate functions, these changes were seen in both the PSE and standard deviation measures. Their group PSE was $538 \mathrm{~ms}$ pre-surgery and $564 \mathrm{~ms}$ post-surgery. The standard deviation score for the lesioned group rose to $239 \mathrm{~ms}$ following surgery from a baseline score of $192 \mathrm{~ms}$ ( $24.4 \%$ increase).

On the LR task, the PSE for the sham group changed from $33.4 \mathrm{sec}$ to 33.4 secs while their standard deviation actually decreased $(8.4 \mathrm{sec}$ to 8.2 $\mathrm{sec})$. The lesion group showed a modest increase in standard deviation from $8.3 \mathrm{sec}$ to $8.6 \mathrm{sec}$ ( $3.8 \%$ increasc). As with the SR task, however, their PSE increased, going from $32.8 \mathrm{sec}$ pre-surgery to $34.2 \mathrm{sec}$ post-surgery.

Since there were not sufficient trials per stimulus duration in each session. we opted against using global measures and developed two trial-bytrial measures, one to reflect a change in bias and one to reflect a change in acuity. First, we established an average presurgery psychometric function. The response on each trial was scored in terms of its difference from the predicted function. Long responses were assigned a value of 100 and short responses were assigned a value of 0 . For our bias measure, we subtracted these numbers ( 100 or 0$)$ from the mean percent long responses for that duration. For example, the mean percent that the animals responded long for the $850 \mathrm{~ms}$ stimulus was 90. When an animal responded long to this stimulus, their bias score for that trial was -10 ; if they responded short. their bias score was 90 . This score was calculated for each trial. If there was no change in performance. the average score would be 0 whereas an increascd likelihood to respond short would yicld a positive score.

A similar procedure was used to obtain a measure of consistency. The only modification was that the difference score was multiplied by -1 for the five longest durations. Again. this procedure was calculated for each trial individually and a mean score was obtained for each animal. As a whole. the higher the mean consistency score. the more consistent is performance (e.g., short durations are responded to as short and long durations are responded to as long). For example. responding long to the $850 \mathrm{~ms}$ stimulus would result in a score of 10 for that trial. whereas responding short would result in a score of -90 . An appealing feature of these measures is that they result in scores of identical units for the two tasks.

Pre- and post-surgery bias and consistency scores were calculated for each animal for both the SR and LR tasks. The mean values for each group are shown in Figure 2. Given that there were only three animals in the sham 
group, the data were analyzed separately for the two groups (and we will not report these results for the shams since no effects reached significance).

The bias and consistency scores were entered into two ANOVAs (one for each measure) with phase (pre vs. post) and task (SR and LR) as factors.

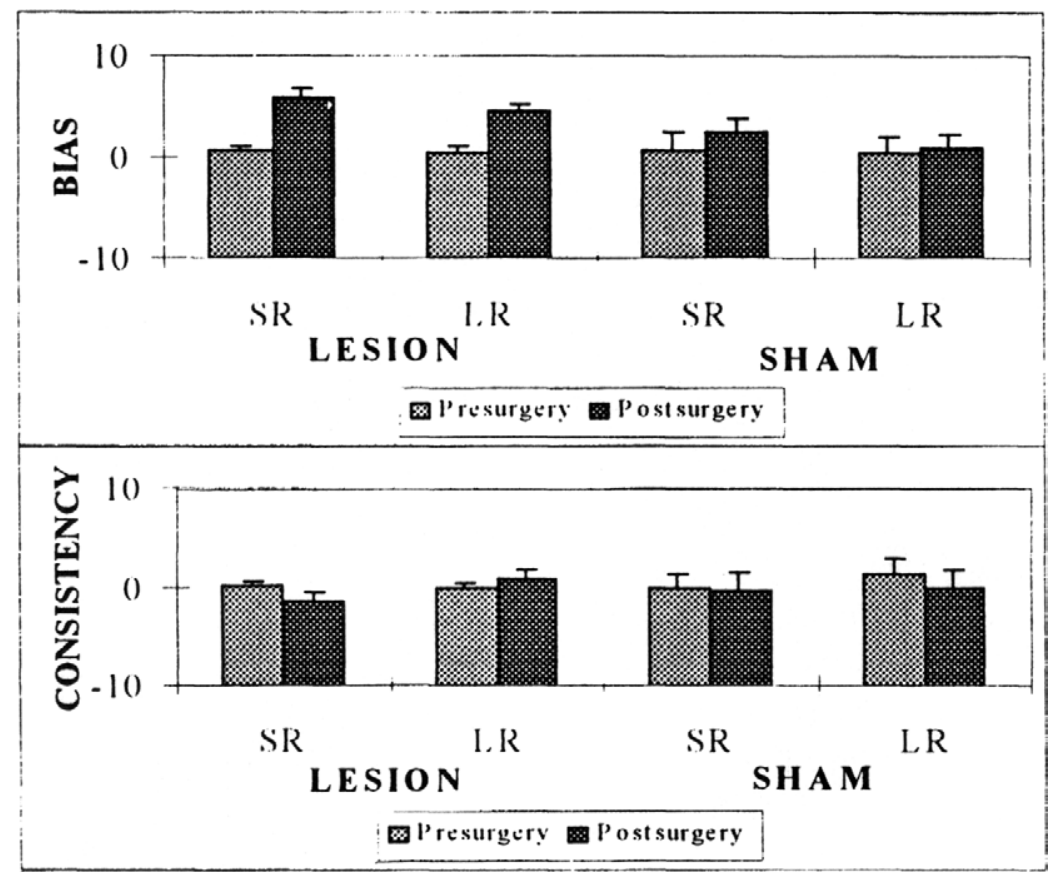

Figure 2: Bias and Consistency scores for Experimemt 1.

On the bias measure. a significant effect was found for phase. $F(1,7)=7.04, p<.05$. The effect of task and the interaction were not significant. Thus, as was seen in the psychometric functions, on both tasks, the lesioned animals were more likcly to respond short following surgery. In contrast. the consistency measure revealed a dissociation between the two tasks. On this measure. both main effects were not significant, but the phase $X$ task interaction was. $F(1.7)=8.40, p<.05$. As can be seen in Figure 2 , when the data are analyzed on a trial-by-trial basis, the animals with cerebellar lesions became less consistent on the SR task following surgery while they became more consistent on the LR task. This result is in accord 
with the hypothesis that the temporal range of the cerebellum is limited to short intervals.

The effect of the lesions was relatively transient. For the first two postsurgery sessions on the SR task, the consistency scores were -5.25 and 4.88. The scores remained greatcr than -1.5 negative for the next three postsurgery sessions, after which they hovered around 0 . We can consider a number of reasons for the transient nature of the deficit. First, histological analyses indicated that our lesions rarely destroyed both lateral nuclei. Moreover, as noted above. it is likely that temporal information is represented in the cercbellar cortex. although the output from cortical neurons would have to pass through the deep nuclei. Second. our animals received many more trials post-surgery than are commonly used in lesion studies. For example. by the fifth post-surgery session on the SR task, the animals had participated in approximately 2,500 trials. This might have been sufficient for reorganization within intact regions of the cerebellum.

\subsection{EXPERIMENT 2}

The dissociation on the consistency measure between the SR and LR tasks was the most positive result in support of the cerebellar timing hypothesis. This finding provides the first evidence of cerebellar involvement on what appears to be a perception of time discrimination in animals. However, there are other aspects of the results that suggest these findings be treated with caution. First. as noted above. the effects were relatively transient and not very large. Second. there was a reliable change in bias for both tasks. While our tasks were designed to equate the response requirements for the two tasks, it is also important to note that the motor demands are not equivalent in the SR and LR tasks. Suppose the animals have to orient after stimulus onset and that cercbellar lesions produce a generalized slowing in this behavior. This deficit would be expected to be more disruptive on the SR task than the LR task because of the brevity of the stimuli in the former condition. If the rats were slow to orient. the brief SR signals might be near completion before the animals have begun to fully attend to the stimulus. In contrast, a slight increase in orienting time would have little effect on the LR task given that the minimum duration was $20 \mathrm{sec}$. The orienting deficit might account for the fact that in both conditions. the lesioned animals were more likely to respond short. 
We have completed a second experiment to test this hypothesis. Two tasks were used in this expcriment: a modified version of the SR task and a non-temporal perceptual task that also involved short signals. For this control task, the rats were required to judge the intensity of a $550 \mathrm{~ms}$ signal. If the cerebellar lesions are disrupting an internal timing system. then the rats should show a selective deficit on the duration discrimination task. If the lesions are producing a gencralized slowing in orienting, then performance on both tasks should be disnupted. The selection of an intensity task was based on our human studies in which we found cerebellar patients to be unimpaired in judging the loudness of brief tones.

4.2.1. Method. Subjects: Twelve new rats were tested in Experiment 2. The animals were housed and fed as in the first experiment.

Apparatus: The same operant boxes were used with one modification. A speaker was mounted above the mesh ceiling in order to present an auditory stimulus consisting of white noise.

Surgery. Surgical procedures were as in the first experiment. The only change was that two penetrations werc made for the lesions on each side. with the second penetration bcing slightly displaced from the first. This change was adopted in an attempt to increase the size of the lesions. Bilateral lesions were performed on nine animals with the remaining three undergoing the sham procedure.

Procedure. There were a number of significant modifications in the procedure for Experiment 2. First. the SR task was modified so that the rats had to judge an empty interval. This modification was adopted to reduce the possibility that the animals were using a non-temporal cue such as energy' integration for the timing task. For cach trial on this task, two $50 \mathrm{~ms}$ markers were presented. separated by an inter-stimulus onset time of the target duration. For the training pliase. the initial intervals were $250 \mathrm{~ms}$ and $1000 \mathrm{~ms}$. After performance asymptoted. these values were changed to 300 and $800 \mathrm{~ms}$. Because the lights in the operant chamber have slow decay rates, the short markers required that we use auditory, white noise stimuli for the $50 \mathrm{~ms}$ markers. In the test phase. nine probe durations were added ranging from $250 \mathrm{~ms}$ to $850 \mathrm{~ms}$.

Second. an intensity discrimination (ID) control task was substituted for the LR task. For this task. a continuous white noise was played for $550 \mathrm{~ms}$ on each trial. For the training phase. the softest and loudest sounds were initially set to intensities of $60 \mathrm{~dB}$ and $80 \mathrm{~dB}$, respectively. These valucs 
were adjusted to $65 \mathrm{~dB}$ and $79 \mathrm{~dB}$, once performance had reached an asymptotic value. The nine probe stimuli ranged from a low of $62 \mathrm{~dB}$ to a high of $82 \mathrm{~dB}$.

Third, all sessions were four hours in duration and alternated by session between the two tasks. We did not include any mixed sessions since an auditory stimulus was used for both tasks. On each day, one group was tested on the SR task and the other group was tested on the ID task. All animals received extensive training on the two tasks prior to surgery. After a one-week recovery period. they were tested for an additional 26 sessions post-surgery. The following analyses are restricted to the last 16 sessions before the operation and the first 16 sessions after the operation. Thus, they include eight sessions per task. pre- and post-surgery.

4.2.2. Results And Discussion. The psychometric functions for the two tasks are presented in Figure 3. For the SR task. the data points correspond to the probability that the animals would respond long for each duration. For the ID task. the data correspond to the probability that a particular intensity was judged loud. Sham operations produced minimal change in performance. On the SR task, PSE and standard deviation values went from $569 \mathrm{~ms}$ and 153 $\mathrm{ms}$ to $567 \mathrm{~ms}$ and $173 \mathrm{~ms}$ (a $13.1 \%$ increase in sd). On the ID task. the PSE was $72.2 \mathrm{~dB}$ presurgery and $72.4 \mathrm{~dB}$ postsurgery. The standard deviation also showed only a slight change increasing to $4.8 \mathrm{~dB}$ from $4.2 \mathrm{~dB}(14.3 \%)$.

In contrast. the psychometric function on the duration discrimination task became considerably flatter for those animals receiving bilateral cerebellar lesions. Correspondingly: the standard deviation for this group showed a large increase (190 ms to $264 \mathrm{~ms}$. a $38.9 \%$ increase). This increase in variability occurred without any change in PSE: for the pre- and postsurgery functions, the PSE estimates are $569 \mathrm{~ms}$ and $567 \mathrm{~ms}$, respectively. Thus. unlike Experiment 1. the effect of the cerebellar lesions was only observed on our measure of variability without any change in bias. At present, we can not account for the discrepent results on the bias measure.

The cerebellar lesions did not affect performance on the intensity discrimination task. The PSE decreased slightly, going from $72.0 \mathrm{~dB}$ to $71.6 \mathrm{~dB}$ while the standard deviation scores were actually slightly lower post-surgery $(5.1 \mathrm{~dB}$ and $4.9 \mathrm{~dB}$ for the pre- and post-surgery functions. respectively). Thus, cerebellar lesions do not produce a generalized impairment in the ability of rats to respond to brief signals. The rats in this 
group were only impaired when they had to judge the duration of these stimuli.

To statistically evaluate these conclusions, we computed bias and consistency scores as in Experiment I (Figure 4). These data were entered

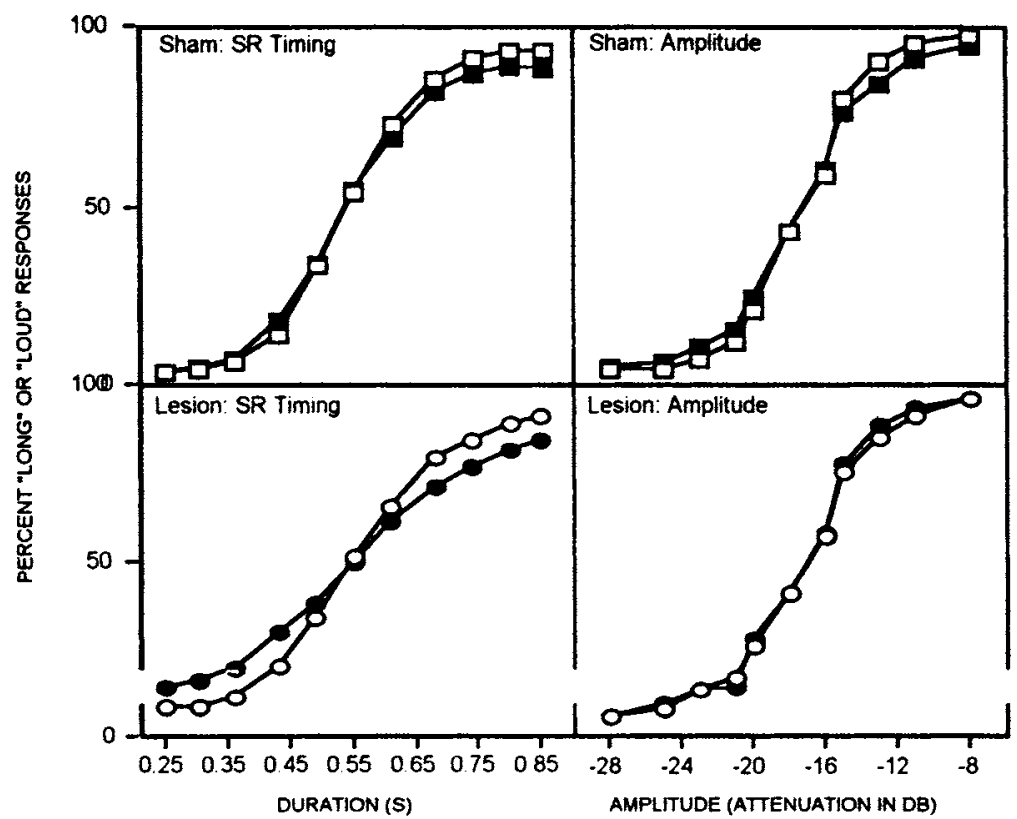

Figure 3: Psychometric functions for Experiment 2. Unfilled symbols represent presurgery performance. filled symbols represent postsurgery performance.

into ANOVAs with two factors: phase (pre- vs. post-surgery) and task (SR vs. ID). No main effects nor interactions were significant for the shams. a result tempered by the fact that there were only three animals in this group. Of greater interest, only the consistency ANOVA yielded significant effects for the cerebellar group. Significant main effects were found for both phase. $F(1.8)=7.24, p<.05$ and task. $F(1.8)=8.87, p<.05$. Furthermore, these main effects were constrained by a significant two-way, phase $\mathrm{X}$ task interaction. $F(1.8)=13.06, p<.01$. A substantial decrease in consistency on the SR task occurred after the animals were given cercbellar lesions. with little change in the ID task. 


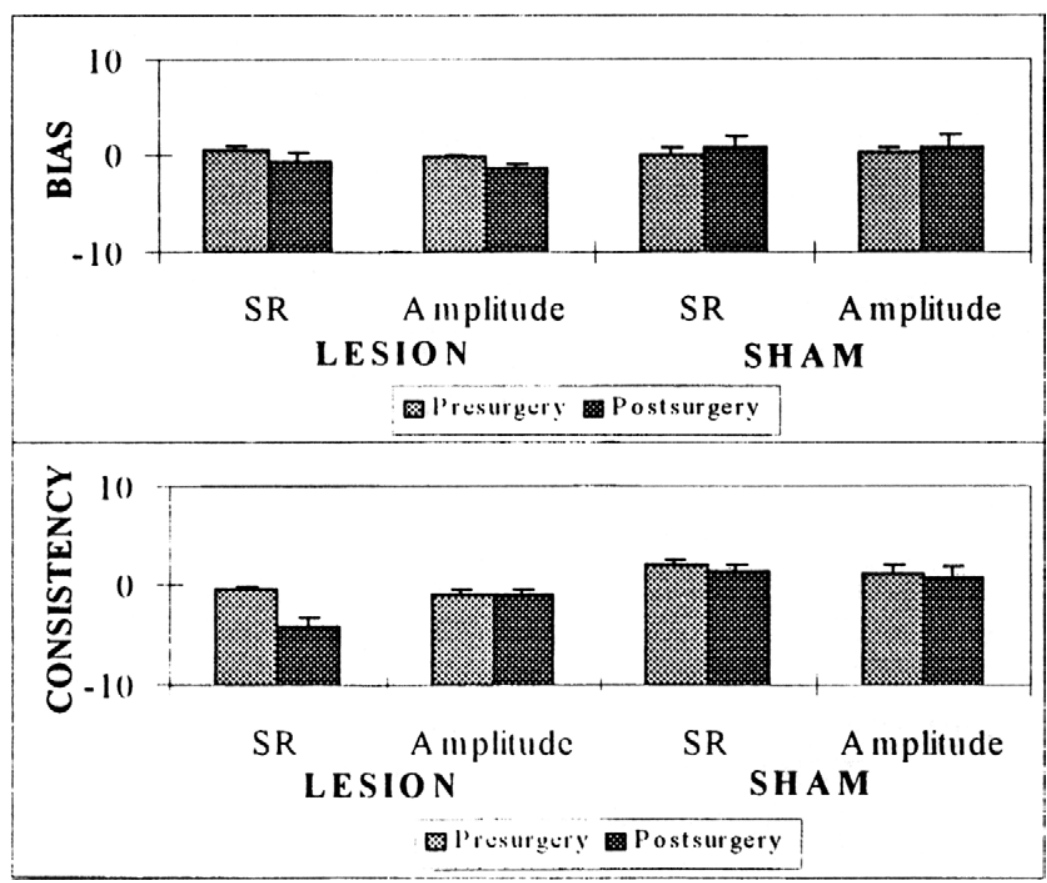

Figure 4: Bias and Consistency data for Experiment 2.

In comparison to Experiment 1 , the nuclear involvement and overall size of the lesions was larger in this experiment. This may have contributed to the fact that across all eight post-surgery sessions on the SR task, the consistency score was at least 1.0 point lower than on the pre-surgery sessions. Nonetheless. the animals showed good recovery with initial mean consistency scores of -6.2 and -8.8 dropping for the first two post-surgery sessions improving to -.17 and -1.8 by the last two sessions. When considering this recovery. it is important to keep in mind that the animals received over 450 trials per session.

\section{Conclusions}

We have proposed that the cercbellum plays a critical role in the representation of temporal information. The initial empirical support for this hypothesis came from research with the study of patients with cerebellar 
lesions (Ivry and Keele. 1989). and included tests of both time production and time perception. To datc, animal studies have continued to focus on how this timing capability may be expressed in motor performance. The current studies provide initial evidence in the rat that the cerebellum may be essential for the perceptual representation of stimulus duration. even when that percept is only indirectly tied to motor responses. These findings emphasize the importance of considering the computational capacity of a particular neural structure when considering its domain. Rather than focus on particular tasks (e.g., reflex conditioning, motor control, perceptual processing). it can be useful to consider the operation performed by a structure and then explore how widely that operation is employed. In the case of the cerebellum. it appears that its timing capability can help account for why this structure is essential for coordinated movement, speech production, sensorimotor learning. as well as certain perceptual functions (see Ivry, 1993).

Although the experimental record is at present quite scant, computational considerations also motivate our conjecture that the temporal extent of the cerebellum is limited to relatively bricf durations. A timing system that is used for coordinating the actions of multiple joints or perceiving the velocity of a moving stimulus is unlikcly to be useful for tasks that span many seconds or minutes. Rescarchers utilizing tasks such as the peak procedure have suggested very different evolutionary pressures for why rats might benefit from being able to determine when $40 \mathrm{sec}$ has elapsed. Such a capability could help the animal determine when the expected return at a particular feeding area should begin to diminish (Roberts, 1983). It is unlikely that this representation would need to have the kind of temporal precision required in skilled movement. Indeed, performance on such tasks may not have the same real-time requirements, perhaps being sufficiently served by inferential mechanisms based on behavioral events (Killeen and Fetterman, 1988) or attentional processes.

\section{References}

Allan LG, Gibbon J. Human bisection at the geometric mean. Learn.. Motiv. 1991: 22 (1-2): 39-58. 
Berger TW. Thompson RF. Neuronal plasticity in the limbic system during classical conditioning of the rabbit nictitating membrane response. I, the hippocampus. Brain Res. 1978: 145 (2): 323-46.

Berger TW, Berry SD. Thompson RF. Role of the hippocampus in classical conditioning of aversive and appetitive behaviors. In: Isaacso RL, Pribram KH, editors. The hippocampus. New York: Plenum Press. 1986: 203-39.

Bullock D. Fiala J. Grossburg S. A ncural model of timed response learning in the cerebellum. Neural Networks. 1994: 7: 1101-14.

Buonomano DV. Mauk MD. Ncural nctwork model of the cerebellum: temporal discrimination and the timing of motor responses. Neural Comput. 1994; 6: 38-55.

Flaherty CF. Animal learning and cognition. New York: Mcgraw-Hill. 1985.

Fraisse. P. The psychology of timc. Ncw York: Harper. 1963.

Getty DJ. Discrimination of short temporal intervals: a comparison of two models. Percep. Psychophy's. 1975: 18 (1): 1-8.

Gibbon J, Church RM. Sources of variance in an information processing theory of animal timing. In: Roitblat HL. Bever TG, Terrace HS editors. Animal cognition. Hillsdale. NJ: Erlbaum. 1984: 465-88.

Ivry R. Cerebellar involvement in the explicit representation of temporal information. In: Tallal P. Galaburda AM. Llinas RR, von Euler C, editors. Temporal Information Processing in the Nervous System. New York: Ann. N. Y. Acad. Sci. 1943: 682: 214-30.

Ivry RB. Keele SW. Timing functions of the cerebellum. J. Cog. Neurol. 1989; 1 (2): 136-52.

Ivry RB, Diener HC. Impaired velocity perception in patients with lesions of the cerebcllum. J. Cog. Ncurol. 1991: 3: 355-66. 
Ivry RB, Hazeltine RE. Pcrception and production of temporal intervals across a range of durations- evidence for a common timing mechanism. J. Exp. Psych.: Hum. Perc. Perf. 1995; 21 (1): 3-18.

Ivry RB, Keele SW, Dicner HC. Dissociation of the lateral and medial cerebellum in movement timing and movement execution. Exp. Brain Res. 1988; $73: 167-80$.

Jenkins HM. Time and contingency in classical conditioning. In: Gibbon J, Allan L, editors. Timing and time perception. New York: Annals of New York Academy of Sciences. 1984: 423: 242-53.

Keele SW, Ivry R. Does the cercbellum provide a common computation for diverse tasks? In: Diamond A. editor. The development and Neural Bases of Higher Cognitive Functions. New York: Annals of the New York Academy of Sciences. 1991; 608: 179-211.

Keele S. Pokorny R. Corcos D. Ivry' R. Do perception and motor production share common timing mechanisms? Acta Psychol. 1985: 60: 173-93.

Kehoe EJ, Graham-Clarke P, Schreurs BG. Temporal patterns of the rabbit's nictitating membrane response to compound and component stimuli under mixed C.S-US intervals. Bchav. Ncurol. 1989: 103 (2): 283-95.

Killeen PR. Fetterman JG. A bchavioral theory of timing. Psychiol. Rev. 1988; 95 (2): 274-95.

Lavond DG, Lincoln JS. McComick DA. Thompson RF. Effect of bilateral lesions of the dentate and interpositus cercbellar nuclei on conditioning of the heart-rate and nictitating membrane/eyclid responses in the rabbit. Brain Res. 1984: 305 (2): 323-30.

Mates J, Radil T. Muller U. and Poppel E. Temporal integration in sensorimotor sychronization. J. Cog. Neurol. 1994; 6 (4): 332-40.

McCormick DA. Thompson RF. Neuronal responses of the rabbit cerebellum during acquisition and performance of a classically conditioned nictitating membrance-eyclid response. J. Neurol. 1984: 4 (11): 2811-22. 
Meck WH. Selective adjustment of the speed of internal clock and memory processes. J. Exp. Psych.: Animal Bchav. Proc. 1983; 9 (2): 171-201.

Meck WH. Modality-specific circadian rhythmicities influence mechanisms of attention and memory for interval timing. Learn. Motiv. 1991: 22: 15379 .

Meck WH, Church RM. Cholinergic modulation of the content of temporal memory. Behav. Neurol. 1987; 101 (4): 457-64.

Milner B. Interhemispheric differences in the localization of psychological processes in man. Brit. Med. Bull. 1971; 27: 272-77.

Nichelli $P$. The neuropsychology of human temporal information processing. In: Boiler F, Grafiman J. editors. Handbook of Neuropsychology. Amsterdam: Elsevier Science Pub. 1993: 339-71.

Olton DS. Wenk GL. Church RM. Meck WH. Attention and the frontal cortex as examined by simultaneous temporal processing. Neuropsychologia. 1988: 26 (2): 507-18.

Paxinos G, Watson C. The rat brain in stereotaxic coordinates. New York: Academic Press. Inc. 1986.

Perrett SP. Ruiz BP. and Mauk MD. Cerebellar cortex lesions disnupt learning-dependent timing of conditioned eyelid responses. J. Ncurol. 1993; 13 (4): $1708-18$.

Povel D. Internal representation of simple temporal patterns. J. Exp. Psych.: OP. Human Percep. Perform.. 1981: 7 (1): 3-18.

Roberts S. Isolation of an internal clock. J. Exp. Psych.: Animal Behav. Proc. 1981: 7: 242-268.

Roberts S. Properties and functions of an internal clock. In: Mcllgren RL. editor. Animal Cognition and Bchavior. Amsterdam: North Holland Pub. Company. 1983: 345-85. 
Smith, MC. CS-US Interval and US intensity in classical conditioning of the rabbit's nictitating membrane response. J. Comp. Physio. Psychiol. 1968; 66 (3): 679-87.

Steinmentz JE. Classical nictitating membrane conditioning in rabbits with varying interstimulus intervals and direct activation of cerebellar mossy fibers as the CS. Behav. Brain Res. 1990; 38: 97-108.

Thompson RF. The neurobiology of learning and memory. Science. 1986; 233: $941-47$.

Wing AM. Keele S, Margolin DI. Motor disorder and the timing of repetitive movements. New York Academy of Sciences Conference on Timing and Time Perception: Timing of motor programs and temporal patterns. Ann. New York Acad. Sci.. 1984; 423: 183-92.

Wing AM, Kristofferson, AB. Response delays and the timing of discrete motor responses. Percep. Psychophys. 1973; 4 (1): 5-12.

Yeo $\mathrm{CH}$. Cerebellum and classical conditioning of motor responses. In: Wolpaw JR. Schmidt JT, Vaughan TM, editors. Activity-driven CNS changes in lcarning and development. New York: Annals of the New York Academy of Sciences. 1991: 627: 292-304.

Yeo CH. Hardiman MJ. Glickstein M. Discrete lesions of the cerebellar cortex abolish the classically conditioned nictitating membrane response of the rabbit. Behav. Brain. Res. 1984: 13 (3): 261-66. 